\title{
Physicians with multiple patient complaints: ending our silence
}

\author{
Thomas H Gallagher, ${ }^{1}$ Wendy Levinson ${ }^{2}$
}

1Department of Medicine, University of Washington, Seattle, Washington, USA ${ }^{2}$ Department of Medicine, University of Toronto, Toronto, Ontario, Canada

\section{Correspondence to Dr Thomas H Gallagher, Department of Medicine, University of Washington, 4311-11th Ave NE, Suite 230; Seattle, WA 98015, USA; thomasg@uw.edu}

Received 4 February 2013 Accepted 4 February 2013 Published Online First 11 April 2013

\section{SLinked}

- http://dx.doi.org/10.1136/ bmjqs-2012-001691

To cite: Gallagher TH, Levinson W. BMJ Qual Saf 2013;22:521-524.
Patients are generally pleased with their personal physicians and appreciate positive relationships with them. In the recent Hospital Consumer Assessment of Healthcare Providers and Systems (HCAHPS) survey of patients in nearly 4000 US hospitals, $81 \%$ of patients gave the highest marks possible to their communication with their physicians. ${ }^{1}$ Yet some physicians struggle to interact effectively with patients.

The majority of problematic patient-provider interactions go unnoticed, except by the patient. The physicians do not receive feedback and may be unaware that their patients are dissatisfied. Sometimes patients who have had a poor interaction with their physician file a complaint, either with the healthcare institution or with a regulator such as a state Board of Medicine. Prior research, along with the study by Bismark and colleagues in this issue, ${ }^{2}$ highlight how a small number of physicians are responsible for the majority of patient complaints. ${ }^{3}$ Similar findings have been demonstrated previously for other markers of problematic patient-provider interactions such as malpractice claims, when a small minority of physicians are responsible for the majority of lawsuits. ${ }^{4}$ Given the fact that many patients who are unhappy with the communication with their physicians hesitate to complain, the current data on the prevalence of patient complaints represent the tip of the iceberg. ${ }^{6}$

Some organisations are systematically examining patient complaints to understand and improve providers' communication skills and patient satisfaction. ${ }^{3}$ Furthermore, patient complaints are associated with other measures of gaps in quality of care. ${ }^{7}$ However, historically the medical profession has not taken patient complaints especially seriously. Multiple formal patient complaints or allegations of egregious behaviour about an individual physician are often required before regulators investigate.
The rigorous study by Bismark and colleagues documents just how pervasive and concerning the problem of physicians with recurrent patient complaints is. ${ }^{2}$ Three percent of Australian physicians accounted for $49 \%$ of patient complaints, and $1 \%$ accounted for $25 \%$ of patient complaints. Physicians who accumulated multiple prior complaints were highly likely to experience future complaints. As with prior studies, the problems that generated the complaints included a mixture of patient concerns about clinical care and communication breakdowns. ${ }^{6} 89$ While the study was conducted in Australia, it is very likely that the findings would be similar in North America, the UK and elsewhere.

The profession's reticence to respond to patient complaints is concerning in multiple respects. The failure to detect and remediate patient care breakdowns represents a fundamental breach of patientcentred care. Patients need clinicians and the healthcare system to listen to their concerns and use them to improve care for themselves and other patients. Our lack of response to individual physicians who accumulate multiple complaints demonstrates an insufficient commitment to being a truly self-regulating profession. Through our silence we fail to intervene at an earlier stage to improve the communication skills and the quality of care provided by our colleagues. This silence abandons our colleagues but more importantly the future patients with whom these physicians will interact.

\section{WHAT IS THE ROOT CAUSE OF THE PROBLEM?}

Why do such a small number of physicians accumulate such a large number of patient complaints? The patient safety movement has emphasised that most care breakdowns stem from significant system failures rather than purely individual provider error. ${ }^{10}$ On its face, however, 
the problem of physicians with multiple complaints may appear to be one for which responsibility lies primarily with the individual provider and their deficient interpersonal skills. Yet closer observation suggests the root causes, and effective solutions, to the problem of physicians with multiple patient complaints have strong system components as well.

Physicians are likely well aware of their colleagues who are at high risk for generating multiple patient complaints. Those physicians who do not interact well with patients are also likely to have difficulty in their relationships with colleagues or with other providers. Therefore, physicians have probably directly observed or experienced these colleagues' poor interpersonal skills with team members. Even if physicians have not personally had difficult interactions with a colleague, they may well have had patients who had a negative encounter with the involved provider and told the physician about their experiences.

Despite being aware of which colleagues have deficits, more often than not physicians look the other way rather than confronting the problem directly. There are several reasons why conversations with a colleague about their interpersonal skills can be daunting. Expressing such concerns directly to a colleague could be awkward and unpleasant, and might damage the reputation of the individual raising the issue. Sometimes power dynamics complicates the situation as the physician with the poor communication may be a 'superior' or a senior colleague. Different specialties have their own cultures related to discussing sensitive issues.

We also hesitate to act out of uncertainty about whether anything can be done to remedy the situation, assuming that interpersonal skills are a fixed component of providers' personality and not amenable to change. Often healthcare communities lack effective and non-punitive remediation programmes to support providers' interpersonal skills. Finally, the medical profession does not take communication issues as seriously as other quality problems. For example, we sometimes tolerate clinicians who are rude but have superb technical skills. 'Ignore his bedside manner. He is a good surgeon.'

Our difficulty supporting colleagues whose interpersonal skills are lacking also reflects a failure to apply routine and effective quality improvement tools to communication breakdowns. ${ }^{11}$ Rarely if ever are episodes of substantial patient dissatisfaction subjected to root cause analysis to understand how the breakdown happened and to develop strategies for preventing recurrences. Instead, the care breakdown is handled by the patient relations department, with a focus on apologising and pacifying the patient in the hope that they will not share their negative experience with others. Rather, these complaints should be used to identify whether this is a recurrent problem with a specific physician who needs remediation or whether there is a broader system problem that requires a different type of corrective action. For example, do the complaints reflect mostly an individual physician who is rude to patients? Or is the root cause of the patient complaint long waiting times in the clinic-a problem that is likely to involve several clinicians in combination with system shortcomings.

\section{WHAT HAS THE RESPONSE BEEN IN NORTH AMERICA?}

Understanding and responding to patient complaints have been receiving increasing attention in North America. Medicare and Medicaid have developed comprehensive requirements that healthcare organisations must use when responding to patient grievances, a process that requires a written answer to the patient and an appeals process. ${ }^{12}$ Hickson and colleagues have also developed approaches to monitor patient complaints as an indicator of physician risk of litigation, and to promote remediation for physicians who have accumulated multiple complaints. ${ }^{13}$

Many healthcare organisations have been devoting considerable energy to the related problem of physicians who struggle to behave collegially. The American Medical Association Council on Ethical and Judicial Affairs now recognises the categories of 'inappropriate behaviour' ('conduct that is unwarranted and is reasonably interpreted to be demeaning or offensive') and the more serious category of 'disruptive behaviour' ('any abusive conduct, including sexual or other forms of harassment, or other forms of verbal or nonverbal conduct that harms or intimidates others to the extent that quality of care or patient safety could be compromised'). ${ }^{14}$ As a condition of accreditation, since 2009 The Joint Commission has required that organisations have a policy in place for detecting and responding to disruptive physician behaviour. ${ }^{15}$ While these disruptive physician policies generally address poor behaviour towards other healthcare workers, there is good reason to suspect that physicians who behave disruptively towards other healthcare workers also struggle to have positive relationships with patients.

Related work has also sought to highlight the critical role that the presence or absence of 'Cultures of Respect' can play in delivering safe, high-quality healthcare, and how disrespectful behaviour by physicians is too often tolerated. ${ }^{16}{ }^{17}$ In addition, more organisations are developing remediation programmes to support providers' interpersonal skills. ${ }^{18}$ Yet while some remediation programmes directed towards disruptive physicians are reporting anecdotal successes, the evidence regarding effective remediation strategies for disruptive physicians remains limited. ${ }^{19}$

Contemporary views of medical professionalism suggest that physicians with multiple patient complaints or disruptive behaviour should be seen as an individual physician problem and a system problem. ${ }^{20}$ This contemporary paradigm conceptualises 
professionalism as a team sport, emphasising our obligations towards patients and our responsibilities to the colleagues who surround us. ${ }^{21}$ Emphasising our collective obligations also acknowledges the reality that most current healthcare is delivered not by individual providers but rather by healthcare teams. It is our responsibility to act to improve the care provided by our team and to address colleagues' problems when we see them.

\section{HOW SHOULD WE MOVE FORWARD?}

\section{Ending our silence}

First we should make a commitment to speak up and tell our colleagues about ways they can improve carespecifically related to communication. When we see problematic interpersonal behaviours occur we should say something rather than ignoring it and whispering to other colleagues about what we saw happen. This willingness to provide feedback to our colleagues is but one component of a more proactive approach to identifying our colleagues who generate multiple complaints. When feedback alone is insufficient and patient complaints persist, stronger corrective actions will be required. ${ }^{22}$

An enhanced commitment to support our colleagues in this way does not imply an individual obligation to 'police' one's colleagues. Rather, these efforts should be envisioned as a team responsibility and activity. The exact nature of that team will vary depending on how physician practices are organised and institutional circumstances. Physicians working together in a group practice could naturally constitute such a team, as could colleagues within an academic division or clinical service line. Regardless of the composition of the team, the critical recognition is one of a shared obligation to support one another in having positive patient interactions and working together to identify and provide remediation for physicians struggling in this area.

\section{Act locally}

Second, we should act locally. While state Boards of Medicine and other external regulators clearly have an important role to play in responding to patient complaints, ultimately the locus of responsibility for ensuring positive interactions with patients and supporting colleagues is at the local level. Institutional medical staff, academic departments and clinical units within them are much better positioned than external regulators to detect colleagues with potentially problematic patient interactions and support these clinicians around improvement. ${ }^{13}$

A local focus for efforts to support our colleagues' interpersonal skills does have potential downsides. It can be harder to be objective with colleagues that you know well. In addition, physicians will need to work to develop their skills for providing feedback to one another around interpersonal interactions. Regardless, the potential to intervene early and the understanding of environmental factors that such a local focus provides outweigh these downsides.

\section{Develop better metrics}

While as physicians we are likely aware of most of our colleagues who are struggling to have positive interactions with patients, improved metrics and strategies for giving physicians feedback on their interactions with patients would be beneficial. A common denominator among physicians who behave disruptively or have poor patient interactions is impaired selfawareness of how their behaviour is experienced by those around them. ${ }^{23}$ Existing measures of patient satisfaction are relatively insensitive to individual instances of poor patient-provider interactions. ${ }^{24}$ Refined measures of patient satisfaction that are more sensitive to problematic physician interactions should be developed, and once such measures are created the results should be provided with faster turnaround to physicians.

The challenges of practicing medicine today can be so pressing that physicians may be tempted to keep their heads down and focus solely on the patient in front of them. The Bismark paper highlights the major opportunity that exists to intervene when a physician accumulates more than one patient complaint. Since these physicians are at risk over time for accruing multiple complaints, the early complaints represent warning signs that require action from colleagues to prevent future patient problems. The critical first step is for all of us to begin speaking up when we know that a colleague is struggling in their interactions with patients and with peers.

Acknowledgements Thanks to Kathleen Mazor, EdD for helpful suggestions on the manuscript draft.

Competing interests None.

Funding Agency for Healthcare Research and Quality (R18HS19531, RO1HS16506), Robert Wood Johnson Foundation Investigator Award in Health Policy Research (both to Dr Gallagher).

Provenance and peer review Commissioned; internally peer reviewed.

\section{REFERENCES}

1 Center for Medicare \& Medicaid Services. Summary of HCAHPS Survey Results. Baltimore, Maryland. 2012. http://www. hcahpsonline.org/executive_insight/default.aspx (accessed 13 Jan 2013).

2 Bismark M, Spittal MJ, Gurrin LC, et al. Identification of doctors at risk of recurrent complaints: a national study of health care complaints in Australia. BMJ Qual Saf 2013.

3 Hickson GB, Federspiel CF, Pichert JW, et al. Patient complaints and malpractice risk. JAMA 2002;287: 2951-7.

4 Sloan FA, Mergenhagen PM, Burfield WB, et al. Medical malpractice experience of physicians. Predictable or haphazard? JAMA 1989;262:3291-7.

5 Weycker DA, Jensen GA. Medical malpractice among physicians: who will be sued and who will pay? Health Care Manag Sci 2000;3:269-77.

6 Mazor KM, Roblin DW, Greene SM, et al. Toward patient-centered cancer care: patient perceptions of 
problematic events, impact, and response. J Clin Oncol 2012;30:1784-90.

7 Weissman JS, Schneider EC, Weingart SN, et al. Comparing patient-reported hospital adverse events with medical record review: do patients know something that hospitals do not? Ann Intern Med 2008;149:100-8.

8 Hickson GB, Federspiel CF, Blackford J, et al. Patient complaints and malpractice risk in a regional healthcare center. South Med J 2007;100:791-6.

9 Carroll KN, Cooper WO, Blackford JU, et al. Characteristics of families that complain following pediatric emergency visits. Ambul Pediatr 2005;5:326-31.

10 Institute of Medicine (US) Committee on Quality of Health Care in America. To Err is Human: Building a Safer Health System. Washington, DC: National Academy Press, 2000.

11 Levinson W, Pizzo PA. Patient-physician communication: it's about time. JAMA 2011;305:1802-3.

12 Revisions to interpretive guidelines for Centers for Medicare \& Medicaid Services Hospital Conditions of Participation, 42 CFR 482.13, 2005. http://www.medicare.gov/claims-andappeals/index.html (accessed 19 Feb 2013)

13 Hickson GB, Pichert JW, Webb LE, et al. A complementary approach to promoting professionalism: identifying, measuring, and addressing unprofessional behaviors. Acad Med 2007;82:1040-8.

14 American Medical Association. Opinion E-9.045 'Physicians with disruptive behavior'. Chicago, Illinois. http://www.amaassn.org/ama1/pub/upload/mm/code-medical-ethics/9045a.pdf (accessed 19 Feb 2013)
15 The Joint Commission. Leadership standard LD.03.03.01. 2008. Washington, DC.http://www.jointcommission.org/ standards_information/standards.aspx (accessed 19 Feb 2013)

16 Leape LL, Shore MF, Dienstag JL, et al. Perspective: a culture of respect, part 2: creating a culture of respect. Acad Med 2012;87:853-8.

17 Leape LL, Shore MF, Dienstag JL, et al. Perspective: a culture of respect, part 1: the nature and causes of disrespectful behavior by physicians. Acad Med 2012;87:845-52.

18 Samenow CP, Worley LL, Neufeld R, et al. Transformative learning in a professional development course aimed at addressing disruptive physician behavior: a composite case study. Acad Med 2013;88:117-23.

19 Hauer KE, Ciccone A, Henzel TR, et al. Remediation of the deficiencies of physicians across the continuum from medical school to practice: a thematic review of the literature. Acad Med 2009;84:1822-32.

20 Lesser CS, Lucey CR, Egener B, et al. A behavioral and systems view of professionalism. JAMA 2010;304:2732-7.

21 Bell SK, Delbanco T, Anderson-Shaw L, et al. Accountability for medical error: moving beyond blame to advocacy. Chest 2011;140:519-26.

22 Wachter RM, Pronovost PJ. Balancing 'no blame' with accountability in patient safety. N Engl J Med 2009;361:1401-6.

23 Egener B. Addressing physicians' impaired communication skills. J Gen Intern Med 2008;23:1890-5.

24 Mazor KM, Clauser BE, Field T, et al. A demonstration of the impact of response bias on the results of patient satisfaction surveys. Health Serv Res 2002;37:1403-17. 